\title{
Small angular scale measurements of the cosmic microwave background temperature power spectrum from QUaD
}

\section{Citation}

Friedman, R. B., P. Ade, J. Bock, M. Bowden, M. L. Brown, G. Cahill, P. G. Castro, et al. 2009. "Small angular scale measurements of the cosmic microwave background temperature power spectrum from QUaD." The Astrophysical Journal 700 (2) (July 20): L187-L191. doi:10.1088/0004-637x/700/2/l187.

\section{Published Version}

doi:10.1088/0004-637X/700/2/L187

\section{Permanent link}

http://nrs.harvard.edu/urn-3:HUL.InstRepos:25757768

\section{Terms of Use}

This article was downloaded from Harvard University's DASH repository, and is made available under the terms and conditions applicable to Other Posted Material, as set forth at http:// nrs.harvard.edu/urn-3:HUL.InstRepos:dash.current.terms-of-use\#LAA

\section{Share Your Story}

The Harvard community has made this article openly available.

Please share how this access benefits you. Submit a story.

Accessibility 


\title{
SMALL ANGULAR SCALE MEASUREMENTS OF THE COSMIC MICROWAVE BACKGROUND TEMPERATURE POWER SPECTRUM FROM QUAD
}

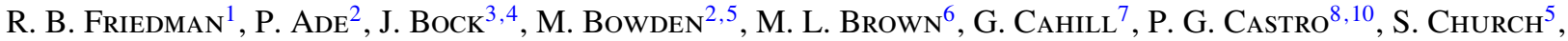 \\ T. Culverhouse ${ }^{1}$, K. Ganga ${ }^{9}$, W. K. Gear ${ }^{2}$, S. Gupta ${ }^{2}$, J. Hinderks $^{5,11}$, J. Kovac ${ }^{4}$, A. E. Lange ${ }^{4}$ E. Leitch $^{3,4}$, \\ S. J. Melhuish ${ }^{12}$, Y. Memari ${ }^{8}$, J. A. Murphy ${ }^{7}$, A. Orlando ${ }^{2,4}$, C. O’ Sullivan ${ }^{7}$, L. Piccirillo ${ }^{12}$, C. Pryke $^{1}$, N. Rajguru $^{2,13}$, \\ B. Rusholme ${ }^{5,14}$, R. Schwarz ${ }^{1}$, A. N. TaYlor ${ }^{8}$, K. L. Thompson ${ }^{5}$, A. H. Turner ${ }^{2}$, E. Y. S. Wu ${ }^{5}$, And M. ZemCOV $^{2,3,4}$ \\ (QUAD COLLABORATION) \\ ${ }^{1}$ Kavli Institute for Cosmological Physics, Department of Astronomy \& Astrophysics, Enrico Fermi Institute, University of Chicago, 5640 South Ellis Avenue, \\ Chicago, IL 60637, USA \\ ${ }^{2}$ School of Physics and Astronomy, Cardiff University, Queen's Buildings, The Parade, Cardiff CF24 3AA, UK \\ 3 Jet Propulsion Laboratory, 4800 Oak Grove Dr., Pasadena, CA 91109, USA \\ ${ }^{4}$ California Institute of Technology, Pasadena, CA 91125, USA \\ ${ }^{5}$ Kavli Institute for Particle Astrophysics and Cosmology, Department of Physics, Stanford University, 382 Via Pueblo Mall, Stanford, CA 94305, USA \\ ${ }^{6}$ Cavendish Laboratory, University of Cambridge, J. J. Thomson Avenue, Cambridge CB3 OHE, UK \\ ${ }^{7}$ Department of Experimental Physics, National University of Ireland Maynooth, Maynooth, Co. Kildare, Republic of Ireland \\ ${ }^{8}$ Institute for Astronomy, University of Edinburgh, Royal Observatory, Blackford Hill, Edinburgh EH9 3HJ, UK \\ ${ }^{9}$ APC/Université Paris 7-Denis Diderot/CNRS, Bâtiment Condorcet, 10, rue Alice Domon et Léonie Duquet, 75205 Paris, Cedex 13, France \\ Received 2009 January 27; accepted 2009 June 26; published 2009 July 20
}

\begin{abstract}
We present measurements of the cosmic microwave background (CMB) radiation temperature anisotropy in the multipole range $2000<\ell<3000$ from the QUaD telescope's second and third observing seasons. After masking the brightest point sources our results are consistent with the primary $\Lambda$ CDM expectation alone. We estimate the contribution of residual (un-masked) radio point sources using a model calibrated to our own bright source observations, and a full simulation of the source finding and masking procedure. Including this contribution slightly improves the $\chi^{2}$. We also fit a standard Sunyaev-Zel'dovich (SZ) template to the bandpowers and see no strong evidence of an SZ contribution, which is as expected for $\sigma_{8} \approx 0.8$.
\end{abstract}

Key words: cosmic microwave background - cosmology: observations

\section{INTRODUCTION}

Observations of the cosmic microwave background (CMB) anisotropy at angular scales of several arcminutes or larger $(\ell<2000)$ have been used to constrain parameters of the $\Lambda \mathrm{CDM}$ cosmological model to high precision (Castro et al. 2009; Dunkley et al. 2009). At these larger angular scales, the anisotropic power is dominated by the primary $\mathrm{CMB}$ from the surface of last scattering. At smaller angular scales $(\ell>2000)$ the primary anisotropy is exponentially suppressed by diffusion in the primordial plasma and the structure becomes dominated by foreground emission and secondary anisotropy generated by intervening large-scale structure. Measuring the secondary anisotropy introduced by the thermal SunyaevZel'dovich effect (SZE) has been of particular interest. The magnitude of the SZE power is a sensitive and independent probe of the amplitude of density perturbations, scaling as $\sigma_{8}^{7}$ (Komatsu \& Seljak 2002).

Previous measurements of the small angular scale $\mathrm{CMB}$ anisotropy at $30 \mathrm{GHz}$ by CBI (Readhead et al. 2004) and BIMA (Dawson et al. 2006) claimed a significant excess over

\footnotetext{
${ }^{10}$ Current address: CENTRA, Departamento de Física, Edifício Ciência, Piso 4, Instituto Superior Técnico-IST, Universidade Técnica de Lisboa, Av. Rovisco Pais 1, 1049-001 Lisboa, Portugal.

${ }^{11}$ Current address: NASA Goddard Space Flight Center, 8800 Greenbelt Road, Greenbelt, MD 20771, USA.

12 Current address: School of Physics and Astronomy, University of Manchester, Manchester M13 9PL, UK.

${ }^{13}$ Current address: Department of Physics and Astronomy, University College London, Gower Street, London WC1E 6BT, UK.

${ }^{14}$ Current address: Infrared Processing and Analysis Center, California Institute of Technology, Pasadena, CA 91125, USA.
}

the $\Lambda \mathrm{CDM}$ expectation at multipoles of $\ell>2000$. The ACBAR experiment (Reichardt et al. 2009) subsequently reported a $\sim 1 \sigma$ excess at $150 \mathrm{GHz}$ at similar scales. Attributing this excess power to the SZE alone implies $\sigma_{8} \approx 1$. This value is in conflict with the WMAP five-year results (Dunkley et al. 2009) and recent $\mathrm{X}$-ray measurements of the cluster mass function (Vikhlinin et al. 2009), which both yield values of $\sigma_{8} \approx 0.8$. For the latter value of $\sigma_{8}$, the SZE power at $30 \mathrm{GHz}$ is expected to be comparable to the primary CMB at multipoles of $\ell \approx 2500$ but at 100 and $150 \mathrm{GHz}$ will be subdominant at multipoles of $\ell<3000$. The results presented in this work cover a multipole range of $2000<\ell<3000$ and are the highest sensitivity to date at these scales.

The QUaD telescope is a millimeter-wavelength bolometric polarimeter located at the South Pole. QUaD operated during the austral winters of 2005 to 2007. Details of the QUaD instrument, calibrations, and performance can be found in Hinderks et al. (2009) and O'Sullivan et al. (2008). In this Letter we present high- $\ell T T$ spectra only. Details of the observations, data quality, low level processing, map-making, and power spectrum estimation plus the full polarization analysis for $\ell<2000$ can be found in Pryke et al. (2009) and Brown et al. (2009) - this Letter follows the analysis methods described there except where noted.

\section{ANALYSIS}

In order to reduce the bandpower uncertainty at high- $\ell$ we have adopted an optimal signal-to-noise Fourier plane weighting step in the power spectrum estimation. As can be seen in Figure 7 of Pryke et al. (2009), the distribution of noise power in 


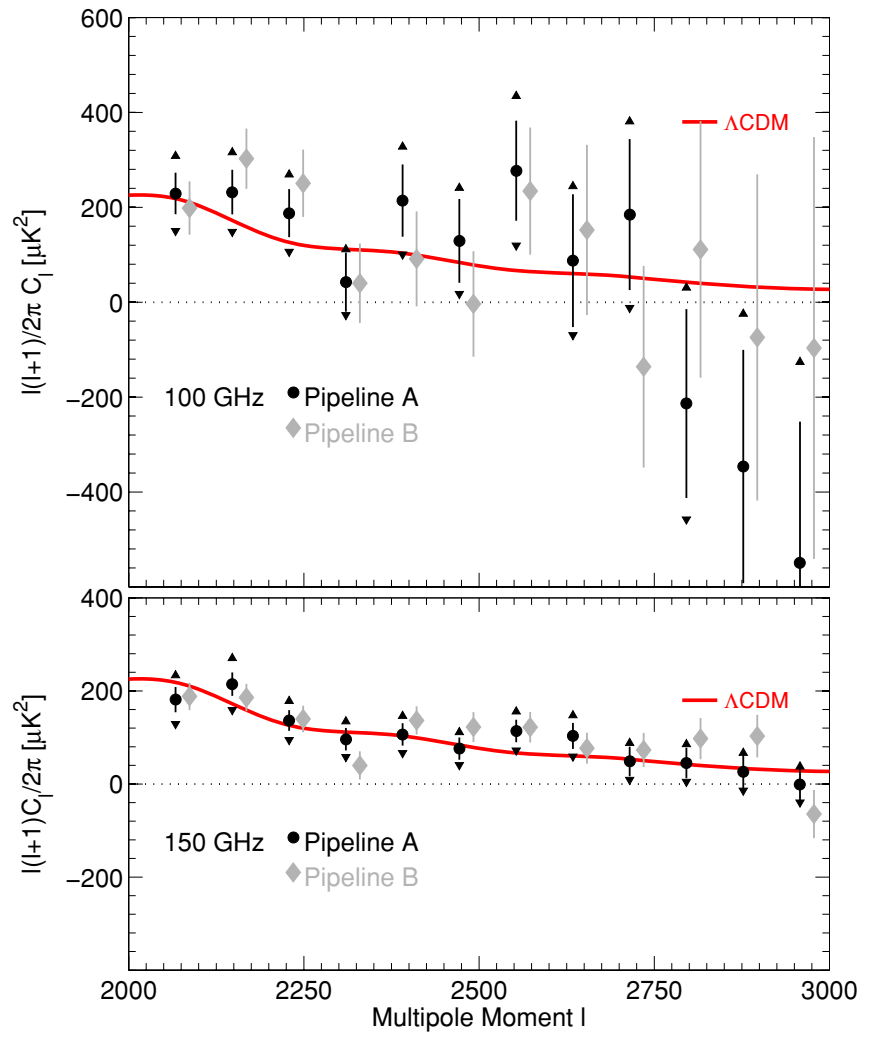

Figure 1. TT bandpower values at $100 \mathrm{GHz}$ (top) and $150 \mathrm{GHz}$ (bottom) vs. $\Lambda C D M$. We show results using the same data from two independent pipelines (see the text); the points are offset for clarity. The error bars include $\Lambda \mathrm{CDM}$ sample variance and noise only. The triangles indicate the coherent shift in the error bar end points which result when the beam and absolute calibration are simultaneously pushed up/down by $1 \sigma$.

the two-dimensional Fourier plane is highly nonuniform-the atmospheric noise forms a concentrated band around the $y$-axis. Fourier plane weights are calculated as

$$
F=\frac{S_{\mathrm{CMB}}^{2}}{\left(S_{\mathrm{CMB}}+N\right)^{2}},
$$

where $S_{\mathrm{CMB}}$ and $N$ are the ensemble averages of the signal and noise simulation two-dimensional auto power spectra. Since the CMB signal is expected to be uniformly distributed in azimuth angle, downweighting localized regions of high noise in the Fourier plane will not bias the results so long as the weighting is independent of the actual data values. This weighting has a dramatic effect on the bandpower uncertainty at high- $\ell$ - for $150 \mathrm{GHz}$ the error is suppressed by as much as an order of magnitude in the range of $2500<\ell<3000$.

In this Letter, we also use two enhancements over the Pryke et al. (2009) analysis introduced in Brown et al. (2009). First, we replace the field differencing operation used to remove ground contamination with a template removal technique that doubles our effective sky area, further reducing the bandpower uncertainties by a factor of $\sim \sqrt{2}$. Second, the beam model used in this Letter has been updated to include sidelobes measured at the $<-20 \mathrm{~dB}$ level and predicted by the physical optics simulations described in O'Sullivan et al. (2008). The absolute calibration uncertainty is now $7 \%$ in power. The cosmological model assumed in our simulations and analysis is the WMAP five-year model given in column 2 of Table 2 in Dunkley et al. (2009) with zero SZE signal, hereafter referred to as $\Lambda$ CDM.

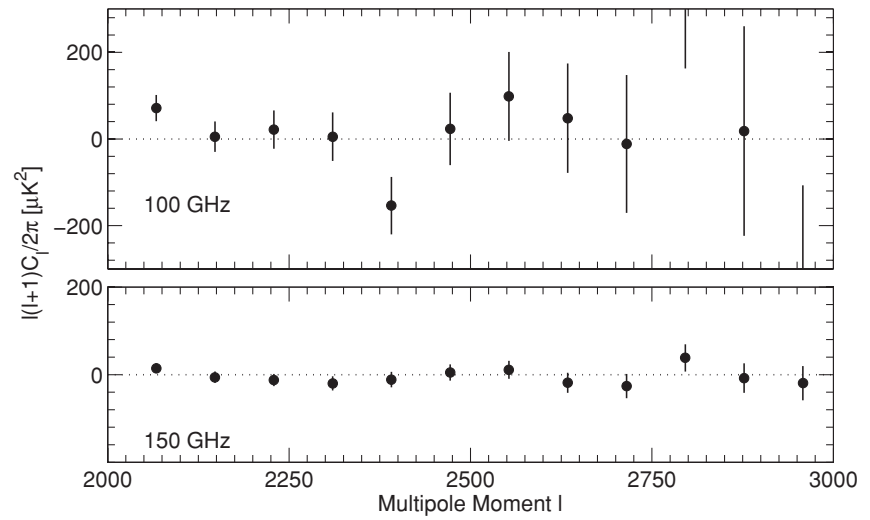

Figure 2. Jackknife $T T$ bandpower values for both the $100 \mathrm{GHz}$ (top) and $150 \mathrm{GHz}$ (bottom) deck-jackknife maps. These spectra are consistent with null and indicate that the signal seen in Figure 1 originates on the sky.

The input simulation spectra are generated using CAMB (Lewis et al. 2000) with lensing turned on.

\section{RESULTS}

In Figure 1 we present our basic result, the $T T$ band power values at 100 and $150 \mathrm{GHz}$ extending to $\ell=3000$. Pipeline A is the pipeline used in Pryke et al. (2009), while Pipeline B is an alternate curved sky analysis (see Ade et al. 2008 and Brown et al. 2009). Both pipelines are based broadly on the MASTER analysis technique (Hivon et al. 2002). The bandpower uncertainties are calculated from the spread in signal plus noise simulation bandpowers assuming the input $\Lambda \mathrm{CDM}$ theory spectrum shown.

As discussed in detail in Pryke et al. (2009), jackknife maps made from differencing independent data sets covering the same sky are a powerful test for systematic contamination. In Figure 2, we present bandpower values for the deck-jackknife-likely our most stringent test. These jackknife spectra are consistent with null.

In the multipole range considered for this analysis, the sky power has been suppressed by almost an order of magnitude through beam convolution. Thus, a small misestimate of the beam would result in a large, multipole-dependent systematic shift in the bandpower values. An under(over-)estimate of the beam suppression would result in an under(over-)estimate of our bandpowers. The effect of the systematic uncertainties on our beam model and calibration is illustrated in Figure 1 where we show the result of pushing both up/down simultaneously by $1 \sigma$. While systematic uncertainty is significant at both frequencies, it is not sufficient to qualitatively change the results at $150 \mathrm{GHz}$.

Though it is customary to present the bandpower results as in Figure 1, it is arguably more natural to consider them as we do in Figure 3 . We calculate $\chi^{2}$ between the data and the simulation distributions using the spread in the signal plus noise realizations to construct a bandpower covariance matrix (see Pryke et al. 2009; Brown et al. 2009). The resulting $\chi^{2}$ values calculated over the $\ell$ bins presented are shown in Figure 3 .

The bandpowers presented in Figures 1 and 3 were calculated after masking bright point sources in the maps as described in Section 4 . We detect seven point sources at $>5 \sigma(\sim 50 \mathrm{~mJ})$ in both the 100 and $150 \mathrm{GHz}$ maps; all of these were matched with a low-probability of chance-association to PMN (Gregory et al. 1994) or SUMSS (Mauch et al. 2003) radio sources using 


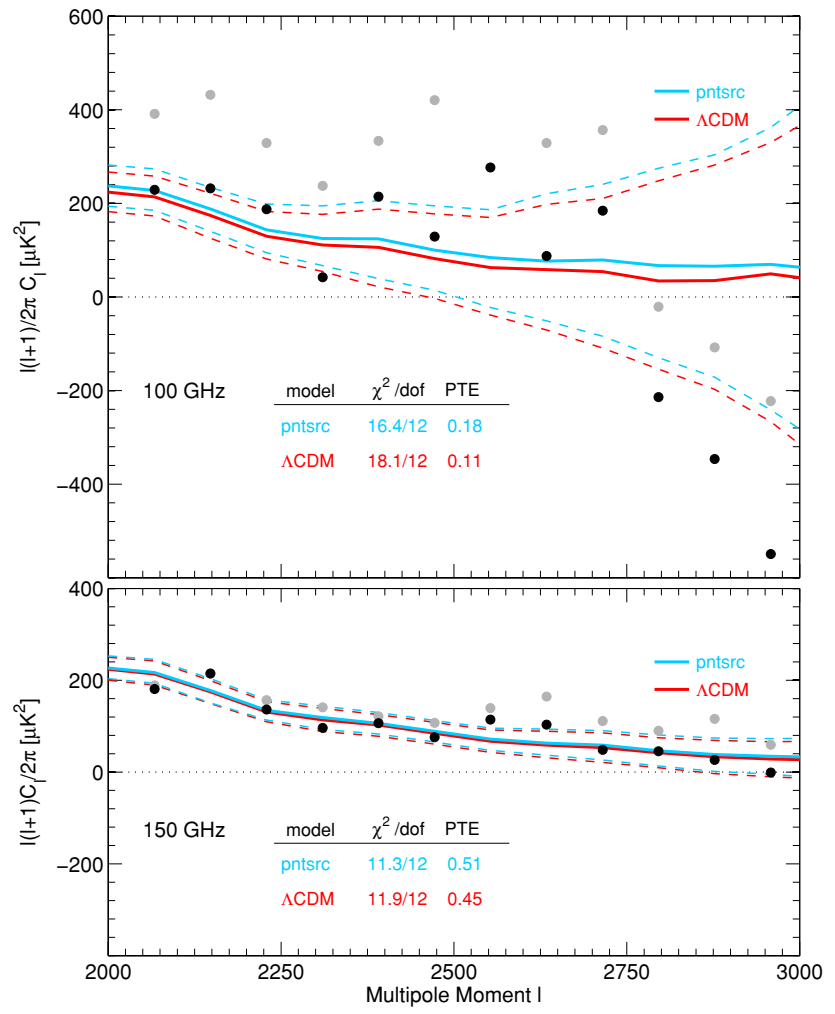

Figure 3. TT bandpower values at $100 \mathrm{GHz}$ (top) and $150 \mathrm{GHz}$ (bottom). This figure shows the same information as Figure 1 but instead of using error bars we plot the spread in the signal plus noise simulations vs. bare points from the data. This is in a sense the fundamental result of the analysis-is the data consistent with being a realization of the simulation model? The lines show the $16 \%, 50 \%$, and $84 \%$ points of the simulation distribution (corresponding to $-1,0,+1 \sigma$ for a Gaussian distribution). Simulation distributions are shown for $\Lambda \mathrm{CDM}$ alone and for $\Lambda \mathrm{CDM}$ plus a residual radio source foreground (pntsrc). The $\chi^{2}$ values are calculated for the data vs. the simulation model (see the text). We also plot bandpowers calculated without masking sources in the maps as light points.

NED. ${ }^{15}$ The effect of masking them can be seen in Figure 3 as the difference between the light and dark points. Since radio source populations typically follow a power-law distribution these sources are only the sparse high flux end of an exponentially more numerous low flux population. It is therefore necessary to estimate the residual power contribution from the unmasked radio source population.

\section{POINT SOURCE SIMULATIONS}

The effect of residual point source contamination in our spectra will manifest as both an increase in the total power at a given $\ell$ and an increase in the bandpower fluctuation. Though it would be straightforward to estimate the mean power contribution from a given point source model and flux cut analytically the subtle effects of source identification and masking in our pipeline would be difficult to account for accurately. Moreover, the fluctuations are potentially nonGaussian. Instead, we explicitly simulate the source population in our maps.

At present, the statistical properties of the sub-Jansky radio source population are not well known at 100 and $150 \mathrm{GHz}$. The most useful published data is the $W$-band $(94 \mathrm{GHz}) W M A P$ point source catalog (Wright et al. 2009). However, this catalog is only complete at fluxes at or above several Jansky whereas even the

\footnotetext{
15 http://nedwww.ipac.caltech.edu/
}

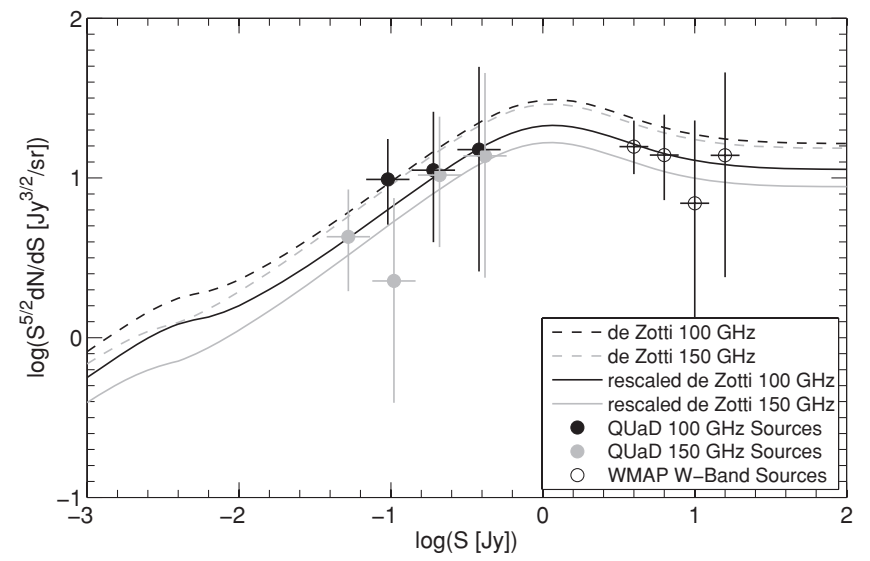

Figure 4. Simulation input point source model (solid lines) is derived by applying a simple rescale factor to the de Zotti radio point source model (dashed lines) (de Zotti et al. 2005) at each frequency. The rescale factor is determined from fits to $d N / d S$ points derived from sources detected in our maps (closed circles). At $100 \mathrm{GHz}$, the rescaling also brings the de Zotti model into better agreement with WMAP W-band $d N / d S$ points (open circles).

brightest sources in the QUaD maps are sub-Jansky. Therefore, to predict the radio source distribution below our detection threshold we use the de Zotti et al. (2005) extragalactic radio source model. Although this is a carefully constructed model using detailed astrophysics, its calibration at lower frequencies makes it a distant extrapolation at $150 \mathrm{GHz}$. We rescale the model by 0.7 and 0.6 at 100 and $150 \mathrm{GHz}$, respectively, to match the number of sources observed in the QUaD maps (see Figure 4). Doing so also brings the model into better agreement with crude $d N / d S$ points determined from the WMAP catalog. We have ignored the possible contribution from IR (dusty) sources.

For point sources, unlike the CMB, we do not simulate the sky signal at the timestream level. Instead, we directly inject source populations into existing signal plus noise maps as beam-sized blips. The fluxes are drawn from the source population model and the positions are uniformly distributed across the map area, i.e., no clustering is assumed.

While sources of low to moderate flux are abundant those of high flux are rare. Leaving the brightest sources in the maps leads to huge contamination as we see comparing the black and gray points in Figure 3. Thus, we must mask sources that are detected at high significance. Given a map containing three components-CMB, noise, and point sources-we need an automated and unbiased method for identifying the point sources to mask.

To separate out the point sources in our maps, we adopt a twodimensional Fourier space optimal filter (Weiner filter) given by

$$
W=\frac{S_{p n t}}{S_{p n t}+S_{\mathrm{CMB}}+N},
$$

where $S_{p n t}$ is the point source signal. We fix $S_{p n t}$ to be a beam-suppressed white-noise power spectrum-representing a uniform distribution of point sources - with an amplitude at $\ell \sim$ 2500 roughly equal to our bandpower value at that multipole. The resulting filters are broadly azimuthally symmetric in form. They are nonzero in the multipole range where we are most sensitive to point source power-i.e., zero at the lowest multipoles where $\mathrm{CMB}$ signal is dominant then rising to a peak near $\ell \sim 2000$ for $100 \mathrm{GHz}$ and $\ell \sim 2500$ for $150 \mathrm{GHz}$ before decaying back to zero at the highest multipoles where instrumental noise is dominant. 


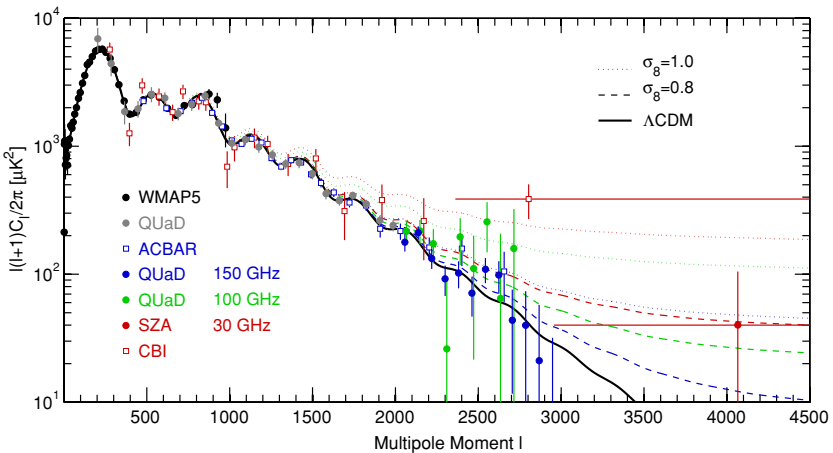

Figure 5. QUaD high- $\ell$ TT results for $2000<\ell<3000$ compared against recen results from ACBAR (Reichardt et al. 2009), CBI (Sievers et al. 2009), and SZA (Sharp et al. 2009)—-spanning the spectral range 30, 100, and $1500 \mathrm{GHz}$ (red, green and blue respectively) - plus WMAP (Hinshaw et al. 2009) (black) and QUaD (Brown et al. 2009) for $\ell<2000$ (gray). For QUaD, SZA, and CBI, the estimated residual radio source contribution has been subtracted. Some points have been slightly offset in multipole for clarity. The data are plotted agains $\Lambda \mathrm{CDM}$ alone and $\Lambda \mathrm{CDM}$ plus the standard Komatsu \& Seljak (2002) template assuming two values of $\sigma_{8}$ scaled to all three frequencies. The QUaD data favors the $\sigma_{8}=0.8$ model with a best-fit scaling of $A_{\mathrm{SZ}}=1.2 \pm 1.2$ (see the text).

The filtered map is then given by

$$
m^{\prime}=\mathrm{FT}\left(W \cdot \mathrm{FT}\left(\frac{m}{v_{\text {pix }}}\right)\right) \cdot v_{\text {pix }},
$$

where FT is the Fourier transform operation, $m$ is the original map, $W$ is the Weiner filter defined in Equation (2), and $v_{\text {pix }}$ is the pixel variance map-used here to appodize $m$-estimated from the timestream rms at the map making stage (see Pryke et al. 2009). For source identification we construct signal-tonoise maps as

$$
s=m^{\prime} v_{\text {pix }}^{-1 / 2} \text {. }
$$

Though the filtering operation changes the noise amplitude, $v_{\text {pix }}$ provides information about the spatial distribution of the noise. We rescale the amplitude of $s$ so that the 16th percentile point of the pixel distribution is equal to -1 . To identify sources in $s$, we subtract a source template-constructed from the backtransform of $W$-from the brightest pixels in $s$ and iterate down to a threshold value of 5 . This procedure results in a source-free $s$-map with pixel values that are close to Gaussian distributed in the range $\pm 5 \sigma$ and a catalog of $>5 \sigma$ sources, where $\sigma \approx 10 \mathrm{mJy}$ at both frequencies.

We generate a source catalog for both the real maps and for each of our point source injected signal plus noise simulated maps and calculate the power spectra masking out the $>5 \sigma$ sources. The resulting distribution of the radio source injected simulations, as compared to $\Lambda \mathrm{CDM}$ alone, is plotted in Figure 3. Comparing the data against this new model we find that the addition of residual radio sources marginally improves the $\chi^{2}$ at both 100 and $150 \mathrm{GHz}$. The bandpower values, together with their covariance matrices and window functions, are available in numerical form at http://quad.uchicago.edu/quad.

\section{IMPLICATIONS FOR SZE FOREGROUNDS}

Figure 5 shows the QUaD High- $\ell$ TT results along with measurements from other recent experiments. There is broad agreement between the various data sets-amongst each other and with $\Lambda \mathrm{CDM}$ - except at the highest multipoles. Here CBI (Sievers et al. 2009) claims a significant excess, which was "confirmed" by ACBAR (Reichardt et al. 2009) at $1 \sigma$, whereas SZA (Sharp et al. 2009) is consistent with zero power. This intimation of excess power has been attributed to SZE signal and used to derive corresponding constraints on the amplitude of density perturbations $\sigma_{8}$. Depending on the template used, the CBI (and to a lesser extent ACBAR) data imply values in the range $0.9<\sigma_{8}<1.0$, a departure from the conventional value of 0.8

Following suit, we add the standard (Komatsu \& Seljak 2002, hereafter KS) template-scaled by a single parameter $A_{\mathrm{SZ}}$ - to $\Lambda \mathrm{CDM}$ plus the residual radio source contribution (Section 4) and fit this model to the QUaD bandpowers. The scale parameter can be related to a value of $\sigma_{8}$ as

$$
\sigma_{8}^{K S} \approx 0.8 A_{\mathrm{SZ}}^{1 / 7}
$$

The 100 and $150 \mathrm{GHz}$ data are considered both independently and simultaneously. We use the bandpower covariance matrix from simulations and add a beam plus absolute calibration systematic term calculated as

$$
\mathrm{M}_{b b^{\prime}}^{\prime}=\mathrm{M}_{b b^{\prime}}+a^{2}\left(S_{b} \hat{C_{b}}\right)\left(S_{b^{\prime}} \hat{C_{b^{\prime}}}\right)
$$

where $\hat{C}_{b}$ is the real bandpower value, $a$ is the absolute calibration uncertainty, and $S_{b}$ is the beam uncertainty. We calculate a $\chi^{2}$ for $A_{\mathrm{SZ}}$ as

$\chi^{2}=\left(\hat{C}_{b}-\left\langle C_{b}^{M C}\right\rangle-A_{\mathrm{SZ}} C_{b}^{K S}\right) \mathrm{M}_{b b^{\prime}}^{\prime-1}\left(\hat{C}_{b^{\prime}}-\left\langle C_{b^{\prime}}^{M C}\right\rangle-A_{\mathrm{SZ}} C_{b^{\prime}}^{K S}\right)$,

where $\hat{C}_{b}, C_{b}^{M C}$, and $C_{b}^{K S}$ are the observed, simulation, and $\mathrm{KS}$-expectation bandpowers, respectively. The sample variance component of the covariance matrix is scaled in proportion to the level of model signal akin to the role of the more conventional offset-lognormal transformation. The resulting likelihood is still very nearly Gaussian.

We note that the procedure adopted here neglects the nonGaussianity of the SZE signal and its fluctuation and to correctly include this would require that we inject simulated SZE sky into our maps; we have not done this.

We quote the maximum-likelihood value, with the $1 \sigma$ uncertainties corresponding to the likelihood falloff that encompasses $68 \%$ of the total; $95 \%$ upper limits are evaluated by integrating the likelihood over positive values of $A_{\mathrm{SZ}}$. The results for 100 and $150 \mathrm{GHz}$ are $A_{\mathrm{SZ}}=0.9 \pm 1.4$ and $1.0 \pm 1.5$ or $A_{\mathrm{SZ}}<3.6$ and 3.8, respectively, with simultaneous fit values of $A_{\mathrm{SZ}}=1.2 \pm 1.2$ or $A_{\mathrm{SZ}}<3.3$.

These results are consistent with SZE power at the expected level for $\sigma_{8}=0.8$ but inconsistent with those of Sievers et al. (2009), preferring lower values of $A_{\mathrm{SZ}}$. There is good agreement with the conclusions of Sharp et al. (2009), who also make a strong argument that the CBI point source estimate is in fact too small. While the frequency dependence of the SZE makes measurements at 100 and $150 \mathrm{GHz}$ intrinsically less sensitive than those at $30 \mathrm{GHz}$, they are also less prone to contamination by radio sources.

\section{CONCLUSIONS}

We have extended the range of the TT-bandpowers from $\mathrm{QUaD}$ to $\ell=3000$ using signal-to-noise weighting to downweight noisy regions of the two-dimensional Fourier plane, an improved method for the removal of ground contamination and higher accuracy beam modeling. After masking point sources detected at high significance in our maps, the spectra are consistent with the $\Lambda$ CDM expectation alone. We have estimated the residual radio source contribution using a physically motivated 
radio source model scaled to fit our bright source counts and find the contribution to be small at $100 \mathrm{GHz}$ and negligible at $150 \mathrm{GHz}$.

A small SZE contribution is expected at $\ell<3000$ for $\sigma_{8}=0.8$ (see Figure 5). Fitting a standard SZE template spectrum to our data results in a best-fit amplitude consistent with the expectation.

We thank Gianfranco De Zotti for providing us with his radio source counts at 100 and $150 \mathrm{GHz}$. QUaD is funded by the National Science Foundation in the USA, through grants AST0096778, ANT-0338138, ANT-0338335, and ANT-0338238, by the UK Science and Technology Facilities Council (STFC) and its predecessor the Particle Physics and Astronomy Research Council (PPARC), and by the Science Foundation Ireland. J.R.H. acknowledges the support of an NSF Graduate Research Fellowship, a Stanford Graduate Fellowship and a NASA Postdoctoral Fellowship. C.P. acknowledges partial support from the Kavli Institute for Cosmological Physics through the grant NSF PHY-0114422. E.Y.W. acknowledges receipt of an NDSEG fellowship. Y.M. acknowledges support from a SUPA Prize studentship. P.G.C. acknowledges funding from the Fundação para a Ciência e a Tecnologia. M.Z. acknowledges support from a NASA Postdoctoral Fellowship.

\section{REFERENCES}

Ade, P., et al. 2008, ApJ, 674, 22

Brown, M. L., et al. 2009, ApJ, submitted, arXiv:0906.1003

Castro, P. G., et al. 2009, ApJ, submitted, arXiv:0901.0810

Dawson, K. S., Holzapfel, W. L., Carlstrom, J. E., Joy, M., \& LaRoque, S. J. 2006, ApJ, 647, 13

de Zotti, G., Ricci, R., Mesa, D., Silva, L., Mazzotta, P., Toffolatti, L., \& González-Nuevo, J. 2005, A\&A, 431, 893

Dunkley, J., et al. 2009, ApJS, 180, 306

Gregory, P. C., Vavasour, J. D., Scott, W. K., \& Condon, J. J. 1994, ApJS, 90, 173

Hinderks, J. R., et al. 2009, ApJ, 692, 1221

Hinshaw, G., et al. 2009, ApJS, 180, 225

Hivon, E., Górski, K. M., Netterfield, C. B., Crill, B. P., Prunet, S., \& Hansen, F. 2002, ApJ, 567, 2

Komatsu, E., \& Seljak, U. 2002, MNRAS, 336, 1256

Lewis, A., Challinor, A., \& Lasenby, A. 2000, Astrophys. J., 538, 473

Mauch, T., Murphy, T., Buttery, H. J., Curran, J., Hunstead, R. W., Piestrzynski, B., Robertson, J. G., \& Sadler, E. M. 2003, MNRAS, 342, 1117

O'Sullivan, C., et al. 2008, Infrared Phys. Technol., 51, 277

Pryke, C., et al. 2009, ApJ, 692, 1247

Readhead, A. C. S., et al. 2004, ApJ, 609, 498

Reichardt, C. L., et al. 2009, ApJ, 694, 1200

Sharp, M. K., et al. 2009, ApJ, submitted, arXiv:0901.4342

Sievers, J. L., et al. 2009, arXiv:0901.4540

Vikhlinin, A., et al. 2009, ApJ, 692, 1060

Wright, E. L., et al. 2009, ApJS, 180, 283 